\title{
Sharp Bounds for Oscillatory Integral Operators with Homogeneous Polynomial Phases
}

\author{
Danqing He ${ }^{*}$, Zuoshunhua Shi ${ }^{\dagger}$
}

\begin{abstract}
We obtain sharp $L^{p}$ bounds for oscillatory integral operators with generic homogeneous polynomial phases in several variables. The phases considered in this paper satisfy the rank one condition which is an important notion introduced by Greenleaf, Pramanik and Tang. Under certain additional assumptions, we can establish sharp damping estimates with critical exponents to prove endpoint $L^{p}$ estimates.
\end{abstract}

Keywords: Oscillatory integral operator; Homogeneous polynomial phase; Rank one condition; Optimal decay

2010 Mathematics Subject Classification: 42B20 47G10

\section{Introduction}

Let $T_{\lambda}$ be an oscillatory integral operator of the form

$$
T_{\lambda} f(x)=\int_{\mathbb{R}^{n_{Y}}} e^{i \lambda S(x, y)} \varphi(x, y) f(y) d y, \quad x \in \mathbb{R}^{n_{X}},
$$

where $n_{X}, n_{Y}$ are two positive integers, $\lambda$ is a real parameter, $S$ is a real-valued smooth function and $\varphi$ is a smooth cut-off function near the origin in $\mathbb{R}^{n_{X}} \times \mathbb{R}^{n_{Y}}$. We shall refer to $T_{\lambda}$ as an $\left(n_{X}+n_{Y}\right)$-dimensional oscillatory integral operator. In this paper, our purpose is to establish sharp $L^{p}$ bounds for these operators with homogeneous polynomial phases.

In the $(1+1)$-dimensional setting, Phong and Stein [16] proved the remarkable theorem that the sharp $L^{2}$ decay estimate of $T_{\lambda}$ is determined by the Newton polyhedron of the real -analytic phase $S$; for some related results on oscillatory integrals and oscillatory integral operators see [30, 12, 14, 15, 20, 6, 4]. This result was extended to the case of smooth phases by Greenblatt [7]; see Rychkov [19] for a partial result. On the other hand, estimates of $T_{\lambda}$ on $L^{p}$ were also studied by many authors [9, 18, 28, 29, 11, 23, 21, 22, 5]. Recently, general sharp $L^{p}$ decay estimates have been proved by Xiao [26]. For a survey on degenerate oscillatory and Fourier integral operators, we refer the reader to Greenleaf-Seeger [10].

It is difficult to generalize all of the one dimensional results to general higher dimensional cases. However, some uniform estimates were also obtained with non-sharp decay rates; see

\footnotetext{
${ }^{*}$ Department of Mathematics, Sun Yat-sen (Zhongshan) University, Guangzhou, 510275, P.R. China. E-mail address: hedanqing@mail.sysu.edu.cn.

${ }^{\dagger}$ School of Mathematics and Statistics, Central South University, Changsha, People’s Republic of China. Email address: shizsh@csu.edu.cn.
} 
[1, 2, 3, 18. Greenleaf, Pramanik and Tang [8] introduced the important notion of rank one condition to establish sharp $L^{2}$ estimates when the phases are generic homogeneous polynomials; for a earlier result in $(2+1)$ dimensions, see [25]. Under the rank one condition, we shall extend the $L^{2}$ result in Greenleaf-Pramanik-Tang [8] to the $L^{p}$ setting in this paper. In this direction, $\mathrm{Xu}$ and Yan [27] considered the special case $n_{X}=n_{Y}$ and obtained sharp $L^{p}$ estimates for $T_{\lambda}$.

In Section 2, we will prepare some basic tools for our argument. Sharp $L^{p}$ estimates and endpoint estimates will be given in Sections 3 and 4 . For two real numbers $a$ and $b$, we use $a \lesssim b$ to mean $a \leq C b$ for some constant $C>0$, and $a \approx b$ to mean $a \lesssim b$ and $b \lesssim a$. For a linear operator $T$, the notation $\|T\|_{p}$ denotes its operator norm on $L^{p}$.

\section{Preliminaries}

In this section, we shall establish some basic lemmas. A basic property of polynomials will be needed in our application of the operator van der Corput lemma below. The first part of the following lemma is previously known; see Phong-Stein [15, 17].

Lemma 2.1 Assume $P$ is a polynomial in $\mathbb{R}$ with degree not greater than $d$. Then there exists a constant $C=C(d)$, depending only on $d$, such that for any bounded interval $I \subseteq \mathbb{R}$,

$$
\sum_{k=0}^{d}\left|I^{*}\right|^{k} \sup _{x \in I^{*}}\left|P^{(k)}(x)\right| \leq C \sup _{x \in I}|P(x)|,
$$

where $I^{*}$ is the interval with the same center as $I$ but with twice the length of $I$.

Moreover, assume further $P$ is real-valued. If, in addition, there exist two numbers $\mu>$ $0, A>0$ and a bounded interval $J$ such that $\mu \leq|P(x)| \leq A \mu$ for all $x \in J$, then for all $z \in \mathbb{C}$ and all intervals $I \subseteq J, Q_{z}(x)=|P(x)|^{z}$ also satisfies the above estimate with a constant $C=C(d, A, z)$ and $I^{*}$ replaced by $I^{*} \cap J$.

Proof. By translation and scaling, we may assume $I=[0,1]$. Let $V$ be the space of all polynomials in $\mathbb{R}$ with degree not greater than $d$. Then $V$ is a finite-dimensional vector space. It is clear that both sides of (2.2) are norms on $V$. Hence the desired estimate follows.

By our assumption, $P$ has fixed sign on the interval $J$. For example, assume $P>0$. By induction, we can show that all derivatives of $Q_{z}$ have the following form:

$$
\frac{d^{N}}{d x^{N}} Q_{z}(x)=|P(x)|^{z-N} \sum C_{k_{1}, k_{2}, \cdots, k_{N}}(z) P^{\left(k_{1}\right)}(x) P^{\left(k_{2}\right)}(x) \cdots P^{\left(k_{N}\right)}(x)
$$

where the summation is taken over all integers $k_{i} \geq 0$ satisfying $k_{1}+k_{2}+\cdots+k_{N}=N$. For any interval $I \subseteq J$, we can apply the inequality (2.2) to obtain the desired estimate.

Now we give the operator van der Corput lemma due to Phong-Stein [15, 16] and PhongStein-Sturm [18.

The crucial notion of curved trapezoid is given as follows.

Definition 2.1 If $g$ and $h$ are two monotone functions on an interval $[a, b]$, then

$$
\Omega=\{(x, y) \mid a \leq x \leq b, g(x) \leq y \leq h(x)\}
$$

is said to be a curved trapezoid. 
Lemma 2.2 Let $T_{\lambda}$ be a (1+1)-dimensional oscillatory integral operator as in (1.1), where $S$ is a real-valued polynomial in $\mathbb{R}^{2}$ and $\varphi$ is supported in a curved trapezoid $\Omega$. If the Hessian of the phase satisfies $\mu \leq\left|S_{x y}^{\prime \prime}(x, y)\right| \leq A \mu$ on $\Omega$ for two positive numbers $\mu, A>0$, then there exists a constant $C=C(\operatorname{deg}(S), A)$ such that

$$
\left\|T_{\lambda} f\right\|_{L^{2}} \leq C\left(\sum_{k=0}^{2} \sup _{\Omega}\left(\delta_{\Omega, h}(x)\right)^{k}\left|\partial_{y}^{k} \varphi(x, y)\right|\right)|\lambda \mu|^{-1 / 2}\|f\|_{L^{2}},
$$

where $\delta_{\Omega, h}(x)$ denotes the length of the vertical cross-section $I_{\Omega, h}(x)=\{y \mid(x, y) \in \Omega\}$.

The following simple version of almost orthogonality principle will be frequently used in this paper; see Phong-Stein-Sturm [18] for its proof. For a general set $A$, the notation $\chi_{A}$ denotes the characteristic function of $A$.

Lemma 2.3 Let $K$ be a Lebesgue measurable function in $\mathbb{R}^{n_{X}} \times \mathbb{R}^{n_{Y}}$ and $m_{d}$ the Lebesgue measure on $\mathbb{R}^{d}$. Assume that there are measurable sets $A_{i} \subseteq \mathbb{R}^{n_{X}}$ and $B_{i} \subseteq \mathbb{R}^{n_{Y}}$ such that $m_{n_{X}}\left(A_{i} \cap A_{j}\right)=0$ and $m_{n_{Y}}\left(B_{i} \cap B_{j}\right)=0$ for $|i-j| \geq N_{0}$. Let $K_{i}(x, y)=K(x, y) \chi_{A_{i}}(x) \chi_{B_{i}}(y)$ and $T$ (respectively, $T_{i}$ ) be the integral operator associated with the kernel $K$ (respectively, $K_{i}$ ). If $T=\sum_{i} T_{i}$, then for all $1 \leq p \leq \infty$ we have

$$
\|T\|_{p} \leq N_{0} \sup _{i}\left\|T_{i}\right\|_{p}
$$

where $\|T\|_{p}$ and $\left\|T_{i}\right\|_{p}$ denote the operator norms of $T$ and $T_{i}$ respectively, as operators from $L^{p}\left(\mathbb{R}^{n_{Y}}\right)$ into $L^{p}\left(\mathbb{R}^{n_{X}}\right)$.

As a useful interpolation technique, we also need the following interpolation with change of power weights. An earlier version of this lemma appeared in Pan, Sampson and Szeptycki [13] see also [23, 21].

Lemma 2.4 Let $T$ be a sublinear operator mapping simple functions in $\mathbb{R}^{n_{Y}}$, defined with respect to Lebesgue measure, into measurable functions in $\mathbb{R}^{n_{X}}$. Assume that there are constants $A, B>$ 0 and $a \neq-\frac{1}{2 n_{X}}$ such that, for all simple functions in $\mathbb{R}^{n_{Y}}$,

(i) $\|T f\|_{L^{\infty}(d x)} \leq A\|f\|_{L^{1}(d y)}$;

(ii) $\left\||x|^{a} T f\right\|_{L^{2}(d x)} \leq B\|f\|_{L^{2}(d y)}$.

Then for any $\theta \in(0,1)$, there exists a constant $C=C\left(a, n_{X}, \theta\right)$ such that

$$
\left\||x|^{a \theta-(1-\theta) n_{X}} T f\right\|_{L^{p}(d x)} \leq C A^{1-\theta} B^{\theta}\|f\|_{L^{p}(d y)}, \quad \frac{1}{p}=\frac{\theta}{2}+1-\theta .
$$

Proof. Define a measure $d \mu=|x|^{c} d x$ on $\mathbb{R}^{n_{X}}$ and an operator $W f(x)=|x|^{b} T f(x)$, where $b, c \in \mathbb{R}$ are to be determined. Here we need a simple fact that $|x|^{b}$ belongs to $L^{1, \infty}\left(|x|^{c} d x\right)$ in $\mathbb{R}^{n_{X}}$ if and only if $b+c=-n_{X}$ and $b \neq 0$. Now we first consider $b>0$. For any $\lambda>0,|x|^{b}>\lambda$ is equivalent to $|x|>\lambda^{1 / b}$. Hence

$$
d \mu\left(\left\{x \in \mathbb{R}^{n_{X}}:|x|^{b}>\lambda\right\}\right)=\int_{|x|>\lambda^{1 / b}}|x|^{-n_{X}-b} d x=C\left(n_{X}, b\right) \lambda^{-1} .
$$

Thus our claim is true for $b>0$. Similarly, we can show the claim for $b<0$. 
With the above result, we take $b=2 a+n_{X}$ and $c=-2 a-2 n_{X}$. By Assumptions (i) and (ii), $W$ is bounded from $L^{1}\left(\mathbb{R}^{n_{Y}} ; d y\right)$ and $L^{2}\left(\mathbb{R}^{n_{Y}} ; d y\right)$ into $L^{1, \infty}\left(\mathbb{R}^{n_{X}} ; d \mu\right)$ and $L^{2}\left(\mathbb{R}^{n_{X}} ; d \mu\right)$, respectively. By the Marcinkiewicz interpolation theorem, we see that for any $0<\theta<1$, there exists a constant $C=C\left(a, n_{X}, \theta\right)$ such that

$$
\|W f\|_{L^{p}\left(|x|^{c} d x\right)} \leq C A^{1-\theta} B^{\theta}\|f\|_{L^{p}(d y)}
$$

with $\frac{1}{p}=\theta / 2+1-\theta=1-\theta / 2$. In other words,

$$
\left\||x|^{a \theta-(1-\theta) n_{X}} T f\right\|_{L^{p}(d x)} \leq C A^{1-\theta} B^{\theta}\|f\|_{L^{p}(d y)}
$$

for $\frac{1}{p}=1-\theta / 2$. The proof of the lemma is complete.

\section{Sharp $L^{p}$ estimates}

In this section, we shall establish $L^{p}$ estimates for oscillatory integral operators with homogeneous polynomial phases satisfying the rank one condition. The corresponding endpoint $L^{p}$ estimates will be given in Section 4. Now we first introduce the concept of rank one condition due to Greenleaf, Pramanik and Tang [8].

Definition 3.1 Let $S$ be a homogeneous polynomial in $\mathbb{R}^{n_{X}} \times \mathbb{R}^{n_{Y}}$ with real coefficients. We say that $S$ satisfies the rank one condition if $\operatorname{rank}(\operatorname{Hess}(S)(x, y)) \geq 1$ away from the origin, i.e., the system of equations $\partial_{x_{i}} \partial_{y_{j}} S(x, y)=0$ does not have a solution $(x, y) \in \mathbb{R}^{n_{X}} \times \mathbb{R}^{n_{Y}} \backslash\{(0,0)\}$.

Under the rank one condition, we can state our main result in this section as follows.

Theorem 3.1 Assume $S$ is a homogeneous polynomial in $\mathbb{R}^{n_{X}} \times \mathbb{R}^{n_{Y}}$ with real coefficients and degree $d>n_{X}+n_{Y}$. Let $T_{\lambda}$ be the oscillatory integral operator as in (1.1). If $S$ satisfies the rank one condition, then for $p$ in the following range

$$
\frac{d-n_{Y}+n_{X}}{d-n_{Y}}<p<\frac{d-n_{X}+n_{Y}}{n_{Y}}
$$

there exists a constant $C=C(S, \varphi, p)$ such that

$$
\left\|T_{\lambda} f\right\|_{L^{p}} \leq C|\lambda|^{-\gamma}\|f\|_{L^{p}}, \quad \gamma=\frac{n_{X}}{d} \cdot \frac{1}{p}+\frac{n_{Y}}{d} \cdot \frac{1}{p^{\prime}},
$$

where $p^{\prime}$ is the conjugate exponent of $p$, i.e., $1 / p^{\prime}=1-1 / p$. Moreover, this estimate is sharp provided that the cut-off $\varphi$ does not vanish near the origin.

Proof. Our proof will be divided into two steps.

Step 1. Sharpness of the decay rate.

Assume $\varphi(0,0) \neq 0$ and $|\lambda|$ is sufficiently large. Let $f(y)=\chi_{\left\{|y| \leq \epsilon_{0}|\lambda|^{-1 / d}\right\}}$ for some small $\epsilon_{0}>0$. For $x \in \mathbb{R}^{n_{X}}$ near the origin, $|x| \leq \epsilon_{0}|\lambda|^{-1 / d}$, we obtain $\left|T_{\lambda} f(x)\right| \gtrsim|\lambda|^{-n_{Y} / d}$. Hence

$$
\|T\|_{p} \geq\left\|T_{\lambda} f\right\|_{L^{p}} /\|f\|_{L^{p}} \gtrsim|\lambda|^{-n_{Y} / d}|\lambda|^{-\frac{n_{X}}{d p}} /|\lambda|^{-\frac{n_{Y}}{d p}}=|\lambda|^{-\gamma} .
$$


Step 2. Proof of the optimal decay estimate.

For $r>0$, we use $B_{r}((x, y))$ to denote the ball in $\mathbb{R}^{n_{X}+n_{Y}}$ with radius $r$ and center $(x, y)$. Let $S^{n_{X}+n_{Y}-1}$ be the unit sphere centered at the origin in $\mathbb{R}^{n_{X}+n_{Y}-1}$. In the following argument, we need a partition of unity on $S^{n_{X}+n_{Y}-1}$. For our purpose, we shall first give an appropriate open cover $\left\{\mathcal{O}_{\alpha}\right\}$ of $S^{n_{X}+n_{Y}-1}$.

We first choose a sufficiently small $r>0$ such that for each point $x^{*}:=(x, 0) \in S^{n_{X}+n_{Y}-1}$, there exists a pair of indices $(i, j), 1 \leq i \leq n_{X}, 1 \leq j \leq n_{Y}$, such that the mixed derivative $\partial_{x_{i}} \partial_{x_{j}} S(x, y)$ does not change sign and its absolute value is comparable to a positive constant for all $(x, y) \in B_{r}\left(x^{*}\right) \cap S^{n_{X}+n_{Y}-1}$. Since $S^{n_{X}-1}$ is compact, we can select finitely many points $x_{1}, x_{2}, \cdots, x_{M} \in S^{n_{X}-1}$ such that $\cup_{i} B_{r}\left(x_{i}^{*}\right) \supseteq S^{n_{X}-1} \times\left\{0_{n_{Y}}\right\}$. Here the notation $0_{m}$ denotes the origin in $\mathbb{R}^{m}$. In this way, if $r>0$ is small enough, we can choose $y_{1}, \cdots, y_{N} \in S^{n_{Y}-1}$ such that the union $\cup_{i} B_{r}\left(y_{i}\right)$ covers $S^{n_{Y}-1}$ and some mixed derivative $\partial_{x_{s}} \partial_{y_{t}} S$ does not vanish on each given ball $B_{r}\left(y_{i}^{*}\right)$.

Similarly, there exists a small number $\rho>0$ and finitely many points $w_{1}, w_{2}, \cdots, w_{K} \in$ $S^{n_{X}+n_{Y}-1}$ such that the following three properties hold:

(i) The union of $B_{\rho}\left(w_{k}\right) \cap S^{n_{X}+n_{Y}-1}$ covers the complement of $\cup_{i, j}\left(B_{r}\left(x_{i}^{*}\right) \cup B_{r}\left(y_{j}^{*}\right)\right) \cap$ $S^{n_{X}+n_{Y}-1}$ relative to $S^{n_{X}+n_{Y}-1}$, i.e.,

$$
\bigcup_{k=1}^{K}\left(B_{\rho}\left(w_{k}\right) \cap S^{n_{X}+n_{Y}-1}\right) \supseteq\left(\cup_{i, j}\left(B_{r}\left(x_{i}^{*}\right) \cup B_{r}\left(y_{j}^{*}\right)\right)\right)^{c} \cap S^{n_{X}+n_{Y}-1} ;
$$

(ii) Each $B_{\rho}\left(w_{k}\right)$ does not intersect both $B_{r / 2}\left(x_{i}^{*}\right) \cap S^{n_{X}+n_{Y}-1}$ and $B_{r / 2}\left(y_{j}^{*}\right) \cap S^{n_{X}+n_{Y}-1}$ for all $i$ and $j$, i.e.,

$$
B_{\rho}\left(w_{k}\right) \cap B_{r / 2}\left(x_{i}^{*}\right) \cap S^{n_{X}+n_{Y}-1}=\emptyset, \quad B_{\rho}\left(w_{k}\right) \cap B_{r / 2}\left(y_{j}^{*}\right) \cap S^{n_{X}+n_{Y}-1}=\emptyset ;
$$

(iii) For each $1 \leq k \leq K$, there exists a pair of indices $(s, t)$ such that $\partial_{x_{s}} \partial_{y_{t}} S$ has fixed sign on $B_{\rho}\left(w_{k}\right)$ and its absolute value is bounded from both above and below by positive constants.

Let $\left\{\mathcal{O}_{\alpha}\right\}$ be the open cover consisting of $B_{r}\left(x_{i}^{*}\right) \cap S^{n_{X}+n_{Y}-1}, B_{r}\left(y_{j}^{*}\right) \cap S^{n_{X}+n_{Y}-1}$ and $B_{\rho}\left(w_{k}\right) \cap S^{n_{X}+n_{Y}-1}$. As discussed above, the union of $\mathcal{O}_{\alpha}$ covers the sphere $S^{n_{X}+n_{Y}-1}$. Corresponding to this open cover, we can now construct a partition of unity $\left\{\Psi_{\alpha}\right\}$ such that each $\Psi_{\alpha}$ is homogeneous of degree zero, $\left.\Psi_{\alpha}\right|_{S^{n} X^{+n_{Y}}{ }^{-1}} \in C_{0}^{\infty}\left(\mathcal{O}_{\alpha}\right)$ and $\sum_{\alpha} \Psi_{\alpha}(x)=1$ for all $x \in S^{n_{X}+n_{Y}-1}$.

For each $k \in \mathbb{Z}$ and $\alpha$, we define $T_{\lambda, \alpha, k}$ as $T_{\lambda}$ in (1.1) by insertion of $\Psi_{\alpha}(x, y) \Phi\left(x / 2^{k}, y / 2^{k}\right)$ into the cut-off of $T_{\lambda}$, i.e.,

$$
T_{\lambda, \alpha, k} f(x)=\int_{\mathbb{R}^{n} Y} e^{i \lambda S(x, y)} \Psi_{\alpha}(x, y) \Phi\left(\frac{x}{2^{k}}, \frac{y}{2^{k}}\right) \varphi(x, y) f(d) d y,
$$

where $\Phi \in C_{0}^{\infty}$ is supported in the annulus $1 / 2 \leq|(x, y)| \leq 2$ such that $\sum_{k} \Phi\left(x / 2^{k}, y / 2^{k}\right)=1$ for all $(x, y)$ away from the origin.

In what follows, we shall establish the sharp $L^{p}$ estimate in the theorem. It is more convenient to divide our argument into three cases.

Case $1 \operatorname{supp}\left(\Psi_{\alpha}\right) \cap S^{n_{X}+n_{Y}-1} \subseteq B_{\rho}\left(w_{i}\right)$ for some $i$.

In this case, $\left.\Psi_{\alpha}\right|_{S^{n_{X}}{ }^{+n_{Y}-1}}$ is supported in an open subset of $S^{n_{X}+n_{Y}-1}$ (with subset topology) which does not intersect both the $X$-space and the $Y$-space. Let $T_{\lambda, \alpha}=\sum_{k} T_{\lambda, \alpha, k}$. Then $T_{\lambda, \alpha}$ 
is supported in a cone, with vertex at the origin, which does not intersect both the $X$-space and the $Y$-space away from the origin. Hence for $(x, y)$ in the support of $T_{\lambda, \alpha, k}$, we have $|x| \approx|y| \approx 2^{k}$. It follows immediately that if $|k-l| \geq A_{0}$ for some large positive integer $A_{0}$, then

$$
\mathbb{P}_{X}\left(\operatorname{supp}\left(T_{\lambda, \alpha, k}\right) \cap \operatorname{supp}\left(T_{\lambda, \alpha, l}\right)\right)=\emptyset, \quad \mathbb{P}_{Y}\left(\operatorname{supp}\left(T_{\lambda, \alpha, k}\right) \cap \operatorname{supp}\left(T_{\lambda, \alpha, l}\right)\right)=\emptyset,
$$

where $\mathbb{P}_{X}$ and $\mathbb{P}_{Y}$ are the projections from $\mathbb{R}^{n_{X}+n_{Y}}$ onto the $X$-space $\mathbb{R}^{n_{X}}$ and the $Y$-space $\mathbb{R}^{n_{Y}}$, respectively.

By the almost orthogonality principle in Lemma 2.3, we have $\left\|T_{\lambda, \alpha}\right\|_{p} \leq A_{0} \sup _{k}\left\|T_{\lambda, \alpha, k}\right\|_{p}$ for all $1 \leq p \leq \infty$. To establish our desired estimate, it suffices to prove that each $T_{\lambda, \alpha, k}$ satisfies the $L^{p}$ estimate (3.4).

By our assumption, there exist indices $i_{\alpha}$ and $j_{\alpha}$ such that $\partial_{x_{i_{\alpha}}} \partial_{y_{j_{\alpha}}} S(x, y)$ does not change its sign on the support of $T_{\lambda, \alpha, k}$ and its absolute value is bounded from above and below by positive constants. Hence we can apply Lemma 2.2 with respect to the variables $x_{i_{\alpha}}$ and $y_{j_{\alpha}}$, letting other variables fixed temporarily, and make use of the Schur test, with respect to other variables, to obtain

$$
\begin{aligned}
\left\|T_{\lambda, \alpha, k}\right\|_{2} & \leq C 2^{k\left(n_{X}+n_{Y}-2\right) / 2}\left(|\lambda| 2^{k(d-2)}\right)^{-1 / 2} \\
& =C 2^{k\left(n_{X}+n_{Y}\right) / 2}\left(|\lambda| 2^{k d}\right)^{-1 / 2}
\end{aligned}
$$

On the other hand, since $|x| \approx|y| \approx 2^{k}$ on the support of $T_{\lambda, \alpha, k}$, it is clear that

$$
\left\|T_{\lambda, \alpha, k}\right\|_{1} \leq C 2^{k n_{X}} .
$$

Let $\theta=\frac{2 n_{X}}{d+n_{X}-n_{Y}} \in(0,1)$. With this $\theta$, we use the Riesz-Thörin interpolation theorem to obtain

$$
\left\|T_{\lambda, \alpha, k}\right\|_{p} \leq C|\lambda|^{-\frac{n_{X}}{d+n_{X}-n_{Y}}}
$$

where

$$
\frac{1}{p}=\frac{\theta}{2}+1-\theta=1-\frac{n_{X}}{d+n_{X}-n_{Y}}=\frac{d-n_{Y}}{d+n_{X}-n_{Y}} .
$$

By a duality argument, we also have

$$
\left\|T_{\lambda, \alpha, k}\right\|_{q} \leq C|\lambda|^{-\frac{n_{Y}}{d-n_{X}+n_{Y}}}, \quad q=\frac{d-n_{X}+n_{Y}}{n_{Y}} .
$$

In fact, $T_{\lambda, \alpha, k}$ satisfies the $L^{\infty}$ estimate $\left\|T_{\lambda, \alpha, k}\right\|_{\infty} \leq C 2^{k n_{Y}}$. Interpolation this with the $L^{2}$ inequality (3.6) gives the above $L^{q}$ estimate.

By interpolation, we see that each $T_{\lambda, \alpha, k}$ satisfies the estimate (3.4) uniformly. By the almost orthogonality described as above, we see that $T_{\lambda, \alpha}$ also satisfies the desired estimate.

Case $2 \operatorname{supp}\left(\Psi_{\alpha}\right) \cap S^{n_{X}+n_{Y}-1} \subseteq B_{\rho}\left(x_{i}^{*}\right)$ for some $i$.

In this case, we have $|x| \gtrsim|y|$ and $|x| \approx 2^{k}$ in the support of $T_{\lambda, \alpha, k}$. The almost orthogonality in Case 1 is not true now. By insertion of the damping factor $|x|^{z}$, we shall consider the following damped operator $W_{\lambda, \alpha, k}^{z}$ associated with $T_{\lambda, \alpha, k}$,

$$
W_{\lambda, \alpha, k}^{z} f(x)=\int_{\mathbb{R}^{n_{Y}}} e^{i \lambda S(x, y)} \Psi_{\alpha}(x, y) \Phi\left(\frac{x}{2^{k}}, \frac{y}{2^{k}}\right)|x|^{z} \varphi(x, y) f(d) d y .
$$


Let $W_{\lambda, \alpha}^{z}=\sum_{k} W_{\lambda, \alpha, k}^{z}$. With this definition, it is easy to see that $W_{\lambda, \alpha}^{z}$ is bounded from $L^{1}\left(\mathbb{R}^{n_{Y}}\right)$ into $L^{1, \infty}\left(\mathbb{R}^{n_{X}}\right)$ provided that $z$ has real part $\operatorname{Re}(z)=-n_{X}$.

In what follows, our main purpose is to establish $L^{2}$ damping estimates for $W_{\lambda, \alpha}^{z}$. Let $\sigma_{0}=\frac{d-n_{X}-n_{Y}}{2}$. One will see that $\sigma_{0}$ is the critical exponent in the following damping estimates:

$$
\left\|W_{\lambda, \alpha}^{z}\right\|_{2} \lesssim\left\{\begin{array}{lc}
|\lambda|^{-1 / 2}, & \sigma:=\operatorname{Re}(z)>\sigma_{0} ; \\
|\lambda|^{-1 / 2} \log (2+|\lambda|), & \sigma:=\operatorname{Re}(z)=\sigma_{0} ; \\
|\lambda|^{-\frac{\sigma}{d}-\frac{n_{X}+n_{Y}}{2 d}}, \quad-\min \left\{n_{X}, n_{Y}\right\}<\sigma:=\operatorname{Re}(z)<\sigma_{0} .
\end{array}\right.
$$

In these damping estimates, the implicit constants can take the form $C\left(1+|z|^{2}\right)$ with $C$ independent of $\lambda$ and $z$.

Now we turn to prove (3.10). As in Case 1, we use the operator version van der Corput lemma in Lemma 2.2 to obtain

$$
\left\|W_{\lambda, \alpha, k}^{z}\right\|_{2} \leq C\left(1+|z|^{2}\right)\left(|\lambda| 2^{k d}\right)^{-1 / 2} 2^{k \sigma} 2^{k\left(n_{X}+n_{Y}\right) / 2} .
$$

On the other hand, the size of the support of $W_{\lambda, \alpha, k}^{z}$ implies

$$
\left\|W_{\lambda, \alpha, k}^{z}\right\|_{2} \leq C 2^{k \sigma} 2^{k\left(n_{X}+n_{Y}\right) / 2} .
$$

For $z$ with real part $\sigma$, we have

$$
\begin{aligned}
\left\|W_{\lambda, \alpha}^{z}\right\|_{2} & \lesssim \sum_{k} \min \left\{\left(|\lambda| 2^{k d}\right)^{-1 / 2} 2^{k \sigma} 2^{k\left(n_{X}+n_{Y}\right) / 2}, 2^{k \sigma} 2^{k\left(n_{X}+n_{Y}\right) / 2}\right\} \\
& \lesssim \sum_{2^{k d|\lambda|>1}}\left(|\lambda| 2^{k d}\right)^{-1 / 2} 2^{k \sigma} 2^{k\left(n_{X}+n_{Y}\right) / 2}+\sum_{2^{k d}|\lambda| \leq 1} 2^{k \sigma} 2^{k\left(n_{X}+n_{Y}\right) / 2}
\end{aligned}
$$

If $\sigma>-\min \left\{n_{X}, n_{Y}\right\}$, then it follows from $\sigma+\left(n_{X}+n_{Y}\right) / 2>0$ that the above second summation is bounded by a constant multiple of $|\lambda|^{-\sigma / d-\left(n_{X}+n_{Y}\right) /(2 d)}$. For the first summation, we obtain an upper bound $\lesssim|\lambda|^{-1 / 2}$ for $\sigma>\sigma_{0}$. In the strip $-\min \left\{n_{X}, n_{Y}\right\}<\sigma \leq \sigma_{0}$, the first summation satisfies the same estimate as the second one, up to a logarithmic term for $\sigma=\sigma_{0}$. Combining these estimates, we obtain (3.10).

For $p$ in the range (3.3) and $p \leq 2$, the parameter $\theta$ for which $1 / p=\theta / 2+1-\theta$ must satisfy $\theta_{0}=\frac{2 n_{X}}{d+n_{X}-n_{Y}}<\theta \leq 1$. It should be pointed out that $p$ is just the left endpoint in the interval (3.3) if $\theta=\theta_{0}$. If $-n_{X}(1-\theta)+\sigma \theta=0$ then $0<\sigma<\sigma_{0}$.

Recall that $W_{\lambda, \alpha}^{z}$ is bounded from $L^{1}\left(\mathbb{R}^{n_{Y}}\right)$ into $L^{1, \infty}\left(\mathbb{R}^{n_{X}}\right)$ for $\operatorname{Re}(z)=-n_{X}$. By interpolation in Lemma 2.4, we have

$$
\left\|W_{\lambda, \alpha}^{z}\right\|_{p} \lesssim|\lambda|^{-\left(\frac{\sigma}{d}+\frac{n_{X}+n_{Y}}{2 d}\right) \theta}, \quad \operatorname{Re}(z)=0 .
$$

Here $\theta$ satisfies $1 / p=1-\theta / 2$ and $-n_{X}(1-\theta)+\sigma \theta=0$. Thus $\theta=2 / p^{\prime}$ and $\sigma=n_{X}\left(p^{\prime} / 2-1\right)$ with $p^{\prime}$ being the conjugate exponent of $p$. It follows that the decay exponent above is equal to

$$
\begin{aligned}
\left(\frac{\sigma}{d}+\frac{n_{X}+n_{Y}}{2 d}\right) \theta & =\left[\frac{n_{X}}{d}\left(\frac{p^{\prime}}{2}-1\right)+\frac{n_{X}+n_{Y}}{2 d}\right] \cdot \frac{2}{p^{\prime}} \\
& =\frac{n_{X}}{d p}+\frac{n_{Y}}{d p^{\prime}}
\end{aligned}
$$


as desired.

Case $3 \operatorname{supp}\left(\Psi_{\alpha}\right) \cap S^{n_{X}+n_{Y}-1} \subseteq B_{\rho}\left(y_{j}^{*}\right)$ for some $j$.

In the support of $T_{\lambda, \alpha, k}$, we have $|y| \approx 2^{k}$ and $|y| \gtrsim|x|$. The argument in this case is in many ways like that of Case 2. Define the damped oscillatory integral operator $W_{\lambda, \alpha, k}^{z}$ as in Case 2 with the damping factor $|x|^{z}$ replaced by $|y|^{z}$. As above, $W_{\lambda, \alpha}^{z}=\sum_{k} W_{\lambda, \alpha, k}^{z}$ satisfies the same damping $L^{2}$ estimates. The only difference lies in the situation $\operatorname{Re}(z)=-n_{X}$. In fact, by Fubini's theorem, it is easy to see that $W_{\lambda, \alpha}^{z}$, with $\operatorname{Re}(z)=-n_{X}$, is bounded from $L^{1}\left(\mathbb{R}^{n_{Y}}\right)$ into $L^{1}\left(\mathbb{R}^{n_{X}}\right)$. More precisely, we have

$$
\begin{aligned}
\int_{\mathbb{R}^{n_{X}}}\left|W_{\lambda, \alpha}^{z} f(x)\right| d x & \lesssim \int_{\mathbb{R}^{n_{X}}}\left(\int_{|y| \gtrsim|x|}|y|^{-n_{X}}|f(y)| d y\right) d x \\
& =\int_{\mathbb{R}^{n_{Y}}}|y|^{-n_{X}}|f(y)|\left(\int_{|x| \lesssim|y|} d x\right) d y \\
& \lesssim \int_{\mathbb{R}^{n_{Y}}}|f(y)| d y .
\end{aligned}
$$

By interpolation as in Case 2, $T_{\lambda, \alpha}$ satisfies the desired $L^{p}$ estimate.

Combining all above results, we complete the proof of the theorem.

We can define a class of more general damped oscillatory integral operators associated with $T_{\lambda}$ in (1.1). Let $W_{\lambda, D}^{z}$ be given by

$$
W_{\lambda, D}^{z} f(x)=\int_{\mathbb{R}^{n} Y} e^{i \lambda S(x, y)}|D(x, y)|^{z} \varphi(x, y) f(d) d y
$$

where $z \in \mathbb{C}$ and $D$ is a damping function. Under the rank one condition, we have the following

Theorem 3.2 Assume $S$ is a real-valued homogeneous polynomial in $\mathbb{R}^{n_{X}} \times \mathbb{R}^{n_{Y}}$ with degree $d>n_{X}+n_{Y}$. Let $D$ be a real-valued homogeneous polynomial which does not vanish away from the origin. If $S$ satisfies the rank one condition, then there exists a constant $C=C(S, D, \varphi)$ such that

$$
\left\|W_{\lambda, D}^{z}\right\|_{2} \leq C(1+|z|)^{2} \begin{cases}|\lambda|^{-1 / 2}, & \operatorname{Re}(z)>\frac{d-n_{X}-n_{Y}}{2 d_{D}} ; \\ |\lambda|^{-1 / 2} \log (2+|\lambda|), & \operatorname{Re}(z)=\frac{d-n_{X}-n_{Y}}{2 d_{D}} ; \\ |\lambda|^{-\frac{d_{D}}{d} \operatorname{Re}(z)-\frac{n_{X}+n_{Y}}{2 d},}, & -\frac{\min \left\{n_{X}, n_{Y}\right\}}{d_{D}}<\operatorname{Re}(z)<\frac{d-n_{X}-n_{Y}}{2 d_{D}},\end{cases}
$$

where $d_{D}$ is the degree of $D$.

Remark 3.1 Under the assumptions in the theorem, we can take $D(x, y)=|x|^{2}+|y|^{2}$. Also, the damping function can be chosen as the Hilbert-Schmidt norm of the Hessian of the phase function, i.e.,

$$
D(x, y)=\left(\sum_{i=1}^{n_{X}} \sum_{j=1}^{n_{Y}}\left|\partial_{x_{i}} \partial_{y_{j}} S(x, y)\right|^{2}\right)^{1 / 2} .
$$

Generally, $D$ is not a polynomial but the above damping estimates are still true with $d_{D}=d-2$. In the special case $n_{X}=n_{Y}$, the damping estimates in the theorem, with $D$ being the HilbertSchmidt norm of the Hessian of $S$, were proved by Xu-Yan [27]. For $(1+1)$-dimensional damping estimates with $D=S_{x y}^{\prime \prime}$, we refer the reader to Seeger [20] and Phong-Stein [17]. 
The proof of Theorem 3.2 is the same as that of the damping estimates (3.10). We omit the details here.

\section{Endpoint $L^{p}$ estimates}

Until now, we do not know whether endpoint $L^{p}$ estimates in Theorem 3.1 are true or not. Our proof in Section 3 breaks down since it will produce a logarithmic term. More precisely, we only have

$$
\left\|T_{\lambda} f\right\|_{L^{p}} \leq C|\lambda|^{-\gamma} \log ^{\delta}(2+|\lambda|)\|f\|_{L^{p}}, \quad p \in\left\{\frac{d-n_{Y}+n_{X}}{d-n_{Y}}, \frac{d-n_{X}+n_{Y}}{n_{Y}}\right\},
$$

where $\delta>0$ is a number in $(0,1)$ and $\gamma$ is given by (3.4). In this section, our purpose is to remove this logarithmic term under certain assumptions.

We first introduce a useful notion of nondegeneracy for the phase $S$.

Definition 4.1 Assume $G$ is a continuously differentiable function from $\mathbb{R}^{m}$ into $\mathbb{R}^{n}$. Then $G$ is said to be radially nondegenerate if $\left(x \cdot \nabla_{x}\right) G(x) \neq 0$ for all $x \neq 0$.

Lemma 4.1 Assume $S$ is a homogeneous polynomial in $\mathbb{R}^{n_{X}} \times \mathbb{R}^{n_{Y}}$. Let $P_{i}(x)=\left.\partial_{y_{i}} S(x, y)\right|_{y=0}$ and $Q_{j}(y)=\left.\partial_{x_{j}} S(x, y)\right|_{x=0}$. Then $\nabla_{y} S(x, 0)$ is radially nondegenerate in the $X$-space $\mathbb{R}^{n_{X}}$ if and only if $\left(P_{1}(x), P_{2}(x), \cdots, P_{n_{Y}}(x)\right) \neq 0$ for $x \neq 0$. Similarly, $\nabla_{x} S(0, y)$ is radially nondegenerate in the $Y$-space $\mathbb{R}^{n_{Y}}$ if and only if $\left(Q_{1}(y), Q_{2}(y), \cdots, Q_{n_{X}}(y)\right) \neq 0$ for $y \neq 0$.

Proof. Denote by $d$ the degree of $S$. Since the notion of radial nondegeneracy involves partial derivatives of second order, our assumptions imply that $d \geq 2$ and $P_{i}, Q_{j}$ are homogeneous polynomials of degree $d-1$. By Euler's formula for homogeneous functions,

$$
\left(x \cdot \nabla_{x}\right) \partial_{y_{i}} S(x, 0)=\left(x \cdot \nabla_{x}\right) P_{i}(x)=(d-1) P_{i}(x) .
$$

Similarly, $\left(y \cdot \nabla_{y}\right) \partial_{x_{j}} S(0, y)=(d-1) Q_{j}(y)$. By Definition 4.1, the statement in the lemma follows immediately.

Remark 4.1 In the $X$ and $Y$ spaces, the rank one condition is slightly weaker than the radial nondegeneracy of $\nabla_{y} S(x, 0)$ and $\nabla_{x} S(0, y)$. For example, consider the rank one condition in the $X$-space. Since $y=0$ in the $X$-space, the rank one condition, at the point $(x, 0)$ with $x \neq 0$, implies $\nabla_{x} P_{i}(x) \neq 0$ for some $i$. However, the radial nondegeneracy of $\nabla_{y} S(x, 0)$ is equivalent to $P_{i}(x)=(d-1)^{-1}\left(x \cdot \nabla_{x}\right) P_{i}(x) \neq 0$ for all $i$.

With the concept of radial nondegeneracy, we are able to establish the endpoint $L^{p}$ estimates in Theorem 3.1 .

Theorem 4.2 Assume $S$ is a real-valued homogeneous polynomial with degree $d>n_{X}+n_{Y}$. Suppose $S$ satisfies the following two conditions:

(i) $S$ satisfies the rank one condition in $\mathbb{R}^{n_{X}} \times \mathbb{R}^{n_{Y}}$.

(ii) $\nabla_{y} S(x, 0)$ and $\nabla_{x} S(0, y)$ are radially nondegenerate in $\mathbb{R}^{n_{X}}$ and $\mathbb{R}^{n_{Y}}$, respectively.

Then $T_{\lambda}$ in (1.1) satisfies the $L^{p}$ estimate (3.4) for

$$
\frac{d-n_{Y}+n_{X}}{d-n_{Y}} \leq p \leq \frac{d-n_{X}+n_{Y}}{n_{Y}} .
$$


Moreover, under the assumptions in Theorem 3.2, the damping estimates (3.12) are still true without the logarithmic term $\log (2+|\lambda|)$.

Remark 4.2 There may be no phases satisfying Assumptions (i) and (ii) if $S$ has an even degree. For example, let $n_{X} \geq 2$ and $n_{Y}=1$. Assume $S$ is a homogeneous polynomial and its degree $d$ is even. Then $\partial_{y} S(x, 0)$ is homogeneous in $\mathbb{R}^{n_{X}}$ and its degree is odd. Since $n_{X} \geq 2$, one can see that $\partial_{y} S(x, 0)$ has zeros away from the origin.

However, if $n_{X}=n_{Y}$ or if $d \geq 2$ is odd, then homogeneous phases $S$ satisfying (i) and (ii) always exist. We take two examples, due to Greenleaf, Pramanik and Tang [8], in the following:

(1) If $n_{X}=n_{Y}, S(x, y)=\frac{1}{d-1}\left(\sum_{i=1}^{n_{X}} x_{i}^{d-1} y_{i}+\sum_{i=2}^{n_{X}} x_{i-1} y_{i}^{d-1}+x_{n_{X}} y_{1}^{d-1}\right)$.

(2) Assume $n_{X}>n_{Y}$ and $d \geq 2$ is odd. For example, we can take $S$ as

$$
S(x, y)=\frac{1}{d-1}\left(\sum_{i=1}^{n_{Y}} x_{i}^{d-1} y_{i}+\sum_{i=2}^{n_{Y}} x_{i-1} y_{i}^{d-1}+x_{n_{Y}} y_{1}^{d-1}+\sum_{i=n_{Y}+1}^{n_{X}} \sum_{j=1}^{n_{Y}} x_{i}^{d-1} y_{j}\right) .
$$

Proof. For clarity, we shall divide our proof into two steps. The first step is to prove the $L^{2}$ damping estimates, from which the desired endpoint $L^{p}$ estimates follow immediately.

Step 1. Proof of $L^{2}$ damping estimates.

As in our proof of Theorem 3.1, we shall consider three cases separately. Let $W_{\lambda, D, \alpha, k}^{z}$ be defined as $T_{\lambda, \alpha, k}$ by insertion of a damping factor $|D(x, y)|^{z}$, i.e.,

$$
W_{\lambda, D, \alpha, k}^{z} f(x)=\int_{\mathbb{R}^{n_{Y}}} e^{i \lambda S(x, y)} \Psi_{\alpha}(x, y) \Phi\left(\frac{x}{2^{k}}, \frac{y}{2^{k}}\right)|D(x, y)|^{z} \varphi(x, y) f(d) d y .
$$

Taking summation over $k$, we define $W_{\lambda, D, \alpha}^{z}=\sum_{k} W_{\lambda, D, \alpha, k}^{z}$. Since $W_{\lambda, D, \alpha, k}^{z}=0$ for all $k \gtrsim 1$, we assume $k \lesssim 1$ from now on.

Case $1 \operatorname{supp}\left(\Psi_{\alpha}\right) \cap S^{n_{X}+n_{Y}-1} \subseteq B_{\rho}\left(w_{i}\right)$ for some $i$.

On the support of $W_{\lambda, D, \alpha, k}^{z}$, we have $|D(x, y)| \approx 2^{k d_{D}}$ since $D$ is a homogeneous polynomial which does not vanish away from the origin. Here $d_{D}$ is the degree of $D$. We first apply Lemma 2.2 to two variables $x_{s}$ and $y_{t}$ for which $\partial_{x_{s}} \partial_{y_{t}} S \neq 0$ on the support of $\Psi_{\alpha}$, and then make use of the size estimate to other variables. This will lead to the following estimate:

$$
\begin{aligned}
\left\|W_{\lambda, D, \alpha, k}^{z}\right\|_{2} & \leq C\left(1+|z|^{2}\right)\left(|\lambda| 2^{(k-2) d}\right)^{-1 / 2} 2^{k d_{D} \sigma} 2^{k\left(n_{X}-1\right) / 2} 2^{k\left(n_{Y}-1\right) / 2} \\
& \leq C\left(1+|z|^{2}\right)\left(|\lambda| 2^{k d}\right)^{-1 / 2} 2^{k d_{D} \sigma} 2^{k\left(n_{X}+n_{Y}\right) / 2}
\end{aligned}
$$

where $\sigma:=\operatorname{Re}(z)$. In view of $|x| \approx 2^{k}$ and $|y| \approx 2^{k}$ on the support of $W_{\lambda, D, \alpha, k}^{z}$, the Schur test gives

$$
\left\|W_{\lambda, D, \alpha, k}^{z}\right\|_{2} \leq C 2^{k d_{D} \sigma} 2^{k\left(n_{X}+n_{Y}\right) / 2} .
$$

For $\sigma \geq \frac{d-n_{X}-n_{Y}}{2 d_{D}}$, the exponent of $2^{k}$ is nonnegative in (4.14). This implies $\left\|W_{\lambda, D, \alpha, k}^{z}\right\|_{2} \lesssim$ $|\lambda|^{-1 / 2}$. By Lemma 2.3, as shown in our proof of Theorem 3.1, the desired damping estimate 
holds for $W_{\lambda, D, \alpha}^{z}=\sum_{k} W_{\lambda, D, \alpha, k}^{z}$. For $-\min \left\{n_{X}, n_{Y}\right\} / d_{D}<\sigma<\frac{d-n_{X}-n_{Y}}{2 d_{D}}$, a convex combination of the above two estimates, annihilating the exponent of $2^{k}$, gives the desired estimate.

Case $2 \operatorname{supp}\left(\Psi_{\alpha}\right) \cap S^{n_{X}+n_{Y}-1} \subseteq B_{\rho}\left(x_{i}^{*}\right)$ for some $i$.

The oscillation and size estimates in Case 1 are still true here. However, the almost orthogonality property there does not hold now. As shown in (3.12), we need only show the optimal decay $|\lambda|^{-1 / 2}$ for the critical exponent $\sigma=\frac{d-n_{X}-n_{Y}}{2 d_{D}}$. For this estimate, we claim that there exists a positive number $\delta>0$ such that

$$
\left\|W_{\lambda, D, \alpha, k}^{z} W_{\lambda, D, \alpha, l}^{z *}\right\| \leq C(z)|\lambda|^{-1} 2^{-|k-l| \delta}, \quad \operatorname{Re}(z)=\frac{d-n_{X}-n_{Y}}{2 d_{D}} .
$$

Note that $|x| \approx 2^{k}$ in the support of $W_{\lambda, D, \alpha, k}^{z}$. Hence $W_{\lambda, D, \alpha, k}^{z} W_{\lambda, D, \alpha, l}^{z}=0$ provided that $|k-l|$ is sufficiently large.

To establish (4.16), we shall further impose smallness conditions on the open cover $\left\{\mathcal{O}_{\alpha}\right\}$, constructed in the proof of Theorem 3.1. Choose two open circular cones $U_{\alpha}$ and $V_{\alpha}$, with the same vertex at the origin, such that

(i) $\operatorname{supp}\left(\Psi_{\alpha}\right) \subseteq \overline{U_{\alpha}}$ and $\overline{U_{\alpha}} \backslash\{0\} \subseteq V_{\alpha}$. Here $\overline{U_{\alpha}}$ denotes the closure of $U_{\alpha}$.

(ii) $\operatorname{supp}\left(\Psi_{\alpha}\right), U_{\alpha}$ and $V_{\alpha}$ are so small that for some $i_{\alpha},\left(w \cdot \nabla_{x}\right) \partial_{y_{i_{\alpha}}} S(x, y)$ has fixed sign and does not vanish for all $w \in \mathbb{P}_{X}\left(\overline{U_{\alpha}} \backslash\{0\}\right)$ and $(x, y) \in \overline{U_{\alpha}} \backslash\{0\}$. Here $\mathbb{P}_{X}$ is the projection from $\mathbb{R}^{n_{X}} \times \mathbb{R}^{n_{Y}}$ onto $\mathbb{R}^{n_{X}}$, i.e., $\mathbb{P}_{X}(x, y)=x$.

The assumption (ii) does not lose generality since $\nabla_{y} S(x, 0)$ is radially nondegenerate in the $X$-space. The assumption (i) implies that there exists a large number $N_{\alpha} \geq 1$ such that if $x, x^{\prime} \in \mathbb{P}_{X}\left(U_{\alpha}\right)$ and $|x| /\left|x^{\prime}\right| \geq N_{\alpha}$ then $x-x^{\prime} \in \mathbb{P}_{X}\left(V_{\alpha}\right)$. Combining this observation together the assumption (ii), we obtain

$$
\left|\partial_{y_{i_{\alpha}}} S(x, y)-\partial_{y_{i_{\alpha}}} S(u, y)\right|=\left|(x-u) \cdot \int_{0}^{1} \nabla_{x} \partial_{y_{i_{\alpha}}} S(\theta x+(1-\theta) u, y) d \theta\right| \approx 2^{k(d-1)}
$$

for all $(x, y),(u, y) \in U_{\alpha}$ and $\Phi\left(x / 2^{k}, y / 2^{k}\right) \Phi\left(u / 2^{l}, y / 2^{l}\right) \neq 0$, provided that $|k-l|$, assuming $k \geq l$, is sufficiently large.

In what follows, our purpose is to prove the almost orthogonality estimate (4.16) by the $T T^{*}$ method. First observe that the integral kernel associated with $W_{\lambda, D, \alpha, k}^{z} W_{\lambda, D, \alpha, l}^{z *}$ is given by

$$
\begin{gathered}
K(x, u)=\int_{\mathbb{R}^{n_{Y}}} e^{i \lambda[S(x, y)-S(u, y)]} \Psi_{\alpha}(x, y) \overline{\Psi_{\alpha}(u, y)} \Phi\left(\frac{x}{2^{k}}, \frac{y}{2^{k}}\right) \overline{\Phi\left(\frac{u}{2^{l}}, \frac{y}{2^{l}}\right)} \times \\
|D(x, y)|^{z}|D(u, y)|^{\bar{z}} \varphi(x, y) \overline{\varphi(u, y)} d y .
\end{gathered}
$$

Since $W_{\lambda, D, \alpha, k}^{z} W_{\lambda, D, \alpha, l}^{z}$ and $W_{\lambda, D, \alpha, l}^{z} W_{\lambda, D, \alpha, k}^{z}$ have equal $L^{2}$ operator norms, we can assume $k \geq l$ in the above estimate.

For $f \in C^{1}$, we define a linear differential operator

$$
\mathcal{D} f(y)=\left[i \lambda\left(\partial_{y_{i_{\alpha}}} S(x, y)-\partial_{y_{i_{\alpha}}} S(u, y)\right)\right]^{-1} \partial_{y_{i_{\alpha}}} f(y)
$$


and its transpose $\mathcal{D}^{t}$ by the equality $\int \mathcal{D} f(y) g(y) d y=\int f(y) \mathcal{D}^{t} g(y) d y$ for all $f, g \in C_{c}^{1}$. It is clear that $\mathcal{D} e^{i \lambda[S(x, y)-S(u, y)]}=e^{i \lambda[S(x, y)-S(u, y)]}$. By integration by parts, we have

$$
\begin{gathered}
K(x, u)=\int_{\mathbb{R}^{n_{Y}}} e^{i \lambda[S(x, y)-S(u, y)]} \mathcal{D}^{t}\left(\Psi_{\alpha}(x, y) \overline{\Psi_{\alpha}(u, y)} \Phi\left(\frac{x}{2^{k}}, \frac{y}{2^{k}}\right) \overline{\Phi\left(\frac{u}{2^{l}}, \frac{y}{2^{l}}\right)} \times\right. \\
\left.|D(x, y)|^{z}|D(u, y)|^{\bar{z}} \varphi(x, y) \overline{\varphi(u, y)}\right) d y .
\end{gathered}
$$

The phase function $S(x, y)-S(u, y)$ can be viewed as a polynomial in $y_{i_{\alpha}}$ of degree $\leq d$ with other variables fixed. Without loss of generality, we assume $i_{\alpha}=1$. For arbitrary $x, u \in \mathbb{R}^{n_{X}}$, let $E_{\alpha, k, l}(x, u)$ be the set of $y \in \mathbb{R}^{n_{Y}}$ such that the integrand in (4.18) does not vanish. Take an arbitrary point $y=\left(y_{1}, y^{\prime}\right) \in E_{\alpha, k, l}(x, u)$ with $y^{\prime}=\left(y_{2}, \cdots, y_{n_{Y}}\right) \in \mathbb{R}^{n_{Y}-1}$. If $E_{\alpha, k, l}(x, u)$ is nonempty, then the above assumptions (i) and (ii) imply that as a function of $y_{1}$, with $y^{\prime}$ fixed, $\partial_{y_{1}} S(x, y)-\partial_{y_{1}} S(u, y)$ does not change sign and its absolute value $\approx 2^{k(d-1)}$ on an interval $I$ with length $|I| \approx 2^{l}$. By Lemma 2.1, we have

$$
\begin{aligned}
\sup _{y_{1} \in I}\left|\partial_{y_{1}}\left(\partial_{y_{1}} S(x, y)-\partial_{y_{1}} S(u, y)\right)^{-1}\right| & \lesssim|I|^{-1} 2^{-k(d-1)} \approx 2^{-l} 2^{-k(d-1)}, \\
\left.\sup _{y_{1} \in I}\left|\partial_{y_{1}}\right| D(x, y)\right|^{z} \mid & \lesssim|I|^{-1} 2^{k d_{D} \sigma} \approx 2^{-l} 2^{k d_{D} \sigma} \\
\left.\sup _{y_{1} \in I}\left|\partial_{y_{1}}\right| D(u, y)\right|^{z} \mid & \lesssim|I|^{-1} 2^{k d_{D} \sigma} \approx 2^{-l} 2^{l d_{D} \sigma}
\end{aligned}
$$

where $\sigma=\operatorname{Re}(z)$ and the above implicit constants are independent of $x, u$ and $y^{\prime}$.

Recall that we have assumed $k \geq l$. The $y_{1}$-partial derivative of other cut-off functions in (4.18) is bounded by a constant multiple of $2^{-l}$. Hence we deduce the following pointwise estimate from (4.18):

$$
|K(x, y)| \lesssim\left(|\lambda| 2^{k(d-1)}\right)^{-1} 2^{k d_{D} \sigma} 2^{l d_{D} \sigma} 2^{l\left(n_{Y}-1\right)} \chi_{\left\{|x| \approx 2^{k}\right\}}(x) \chi_{\left\{|u| \approx 2^{l}\right\}}(u) .
$$

Then

$$
\begin{aligned}
\sup _{x} \int_{\mathbb{R}^{n_{X}}}|K(x, u)| d u & \lesssim\left(|\lambda| 2^{k(d-1)}\right)^{-1} 2^{k d_{D} \sigma} 2^{l d_{D} \sigma} 2^{l\left(n_{Y}-1\right)} 2^{l n_{X}}, \\
\sup _{u} \int_{\mathbb{R}^{n_{X}}}|K(x, u)| d x & \lesssim\left(|\lambda| 2^{k(d-1)}\right)^{-1} 2^{k d_{D} \sigma} 2^{l d_{D} \sigma} 2^{l\left(n_{Y}-1\right)} 2^{k n_{X}} .
\end{aligned}
$$

By the Schur test, we obtain

$$
\begin{aligned}
\left\|W_{\lambda, D, \alpha, k}^{z} W_{\lambda, D, \alpha, l}^{z *}\right\|_{2} & \lesssim\left(|\lambda| 2^{k(d-1)}\right)^{-1} 2^{k d_{D} \sigma} 2^{l d_{D} \sigma} 2^{l\left(n_{Y}-1\right)} 2^{(k+l) n_{X} / 2} \\
& \lesssim|\lambda|^{-1} 2^{-|k-l| \delta}, \quad \delta=\frac{d+n_{Y}}{2}-1>0
\end{aligned}
$$

where $\sigma=\operatorname{Re}(z)$ is given by (4.16). By the Cotlar-Knapp-Stein almost orthogonality principle (see Stein [24]), we obtain $\left\|W_{\lambda, D, \alpha}^{z}\right\|_{2} \lesssim|\lambda|^{-1 / 2}$.

Case $3 \operatorname{supp}\left(\Psi_{\alpha}\right) \cap S^{n_{X}+n_{Y}-1} \subseteq B_{\rho}\left(z_{j}^{*}\right)$ for some $j$. 
In this case, the damping estimate with critical damping exponent can be proved as in Case 2 , with the roles of $x$ and $y$ interchanged. The details are omitted here.

Step 2. Proof of the endpoint $L^{p}$ estimates.

In Case $1, W_{\lambda, D, \alpha}^{z}$ satisfies (i) $\left\|W_{\lambda, D, \alpha}^{z}\right\|_{2} \lesssim|\lambda|^{-1 / 2}$ with $\operatorname{Re}(z)=\frac{d-n_{X}-n_{Y}}{2 d_{D}}$ and (ii) $\left\|W_{\lambda, D, \alpha}^{z}\right\|_{1} \lesssim$ 1 with $\operatorname{Re}(z)=-\frac{n_{X}}{d_{D}}$. By interpolation, we obtain the left endpoint $L^{p}$ estimate (3.4).

In Case 2, a slight modification is needed in our argument. We shall replace $D(x, y)$ by $|x|^{d_{D}}$ in the definition of $W_{\lambda, D, \alpha}^{z}$. Then $\left\|W_{\lambda, D, \alpha}^{z}\right\|_{2} \lesssim|\lambda|^{-1 / 2}$ still holds for $\operatorname{Re}(z)=\frac{d-n_{X}-n_{Y}}{2 d_{D}}$. The reason is that $D(x, y)$ and $|x|^{d_{D}}$, together with their partial derivatives, have the same upper bounds in our proof of this critical $L^{2}$ damping estimate. On the other hand, note that $\left\|W_{\lambda, D, \alpha}^{z} f\right\|_{L^{1, \infty}} \lesssim\|f\|_{L^{1}}$ for $\operatorname{Re}(z)=-\frac{n_{X}}{d_{D}}$. By Lemma 2.4, the desired endpoint $L^{p}$ estimate follows.

The Case 3 can be treated in the same way as Case 2. However, we do not need change the damping factor $D$. Now the critical $L^{2}$ damping estimate in Case 2 is still true. For $\operatorname{Re}(z)=-\frac{n_{X}}{d_{D}}$, the stronger estimate $\left\|W_{\lambda, D, \alpha}^{z}\right\|_{1} \lesssim 1$ holds, as in our proof of Theorem 3.1. By a duality argument, we are able to prove the right endpoint $L^{p}$ estimate for (3.4). Thus the proof of the theorem is complete.

Acknowledgements. We would like to thank Shaozhen $\mathrm{Xu}$ for explanation of his work and sharing useful ideas with us.

\section{References}

[1] Carbery, A., Christ, M., Wright, J.: Multidimensional van der Corput and sublevel estimates. J. Amer. Math. Soc., 12, 981-1015 (1999)

[2] Carbery, A., Wright, J. "What is van der Corput's lemma in higher dimensions?" Publicacions Matematiques, 13-26 (2002)

[3] Christ M., Li X., Tao T., Thiele, C.: On multilinear oscillatory integrals, nonsingular and singular. Duke Mathematical Journal, 130, 321-351 (2005)

[4] Gilula M., Some oscillatory integral estimates via real analysis. Mathematische Zeitschrift, 289, 377-403 (2018)

[5] Gilula M., Gressman P. T., Xiao L.: Higher decay inequalities for multilinear oscillatory integrals. Math. Res. Lett., 25, 819-842 (2018)

[6] Greenblatt, M.: A direct resolution of singularities for functions of two variables with applications to analysis. J. Anal. Math., 92, 233-257 (2004)

[7] Greenblatt, M.: Sharp $L^{2}$ estimates for one-dimensional oscillatory integral operators with $C^{\infty}$ phase. Amer. J. Math., 127, 659-695 (2005)

[8] Greenleaf, A., Pramanik, M., Tang, W.: Oscillatory integral operators with homogeneous polynomial phases in several variables. J. Funct. Anal., 244, 444-487 (2007)

[9] Greenleaf, A., Seeger, A.: Oscillatory and Fourier integral operators with folding canonical relations. Studia Math., 132, 125-139 (1999)

[10] Greenleaf, A., Seeger, A.: Oscillatory and Fourier integral operators with degenerate canonical relations. Publ. Mat 93 (2002): 141.

[11] Gressman, P.T., Xiao, L. Maximal decay inequalities for trilinear oscillatory integrals of convolution type. Journal of Functional Analysis, 271, 3695-3726 (2016) 
[12] Hörmander, L.: Oscillatory integrals and multipliers on $F L^{p}$. Ark. Maht. 11, 1-11 (1973)

[13] Pan, Y., Sampson, G., Szeptycki, P.: $L^{2}$ and $L^{p}$ estimates for oscillatory integrals and their extended domains. Studia Math, 122, 201-224 (1997)

[14] Phong, D.H., Stein, E.M.: Oscillatory integrals with polynomial phases. Invent. Math., 110, 39-62 (1992)

[15] Phong, D.H., Stein, E.M.: Models of degenerate Fourier integral operators and Radon transforms. Ann. of Math., 140, 703-722 (1994)

[16] Phong, D.H., Stein, E.M.: The Newton polyhedron and oscillatory integral operators. Acta Math., 179, 105-152 (1997)

[17] Phong, D.H., Stein, E.M.: Damped oscillatory integral operators with analytic phases. Adv. in Math., 134, 146-177 (1998)

[18] Phong, D.H., Stein, E.M., Sturm, J.A.: Multilinear level set operators, oscillatory integral operators, and Newton polyhedra. Mathematische Annalen, 319, 573-596 (2001)

[19] Rychkov, V.S.: Sharp $L^{2}$ bounds for oscillatory integral operators with $C^{\infty}$ phases. Math. Z., 236, 461-489 (2001)

[20] Seeger, A.: Radon transforms and finite type conditions. J. Amer. Math. Soc., 11, 869-897 (1998)

[21] Shi, Z.S.H.: Uniform estimates for oscillatory integral operators with polynomial phases. arXiv preprint arXiv:1809.01300 (2018).

[22] Shi, Z.S.H., Xu, S.Z., Yan, D.Y.: Damping estimates for oscillatory integral operators with real-analytic phases and its applications. To appear in Forum Mathematicum, available online at arXiv preprint arXiv:1809.01298 (2018).

[23] Shi, Z.S.H., Yan, D.Y.: Sharp $L^{p}$-boundedness of oscillatory integral operators with polynomial phases. Mathematische Zeitschrift, 286, 1277-1302 (2017)

[24] Stein, E.M.: Harmonic Analysis: Real variable methods, Orthogonality, and Oscillatory integrals. With the assistance of Timothy S. Murphy, Princeton Univ. Press, 1993. MR $95 \mathrm{c}: 42002$.

[25] Tang, W., Decay rates of oscillatory integral operators in $1+2$ dimensions. Forum Mathematicum, 18, 427-444 (2006)

[26] Xiao, L., Endpoint estimates for one-dimensional oscillatory integral operators. Advances in Mathematics, 316, 255-291 (2017)

[27] Xu, S.Z., Yan, D.Y.: Sharp $L^{p}$ decay of oscillatory integral operators with certain homogeneous polynomial phases in several variables. Science China Mathematics, 62, 649-662 (2019)

[28] Yang, C.W.: Sharp estimates for some oscillatory integral operators on $\mathbb{R}^{1}$. Iillinois Journal of Mathematics, 48, 1093-1103 (2004)

[29] Yang, C.W.: $L^{p}$ improving estimates for some classes of Radon transforms. Trans. Amer. Math. Soc., 357, 3887-3903 (2005)

[30] Varchenko, A.: Newton polyhedra and estimations of oscillatory integrals. Functional Anal. Appl., 18, 175-196 (1976) 